\title{
Adaptive Deterrence Sanctions in a Normative Framework
}

\author{
Henrique Lopes Cardoso, Eugénio Oliveira \\ LIACC, DEI / Faculdade de Engenharia, Universidade do Porto \\ R. Dr. Roberto Frias, 4200-465 Porto, Portugal \\ hlc@fe.up.pt,eco@fe.up.pt
}

\begin{abstract}
Normative environments are used to regulate multiagent interactions. In business encounters, agents representing business entities make contracts including norms that prescribe what agents should do. Agent autonomy, however, gives agents the ability to decide whether they fulfill or violate their commitments. In this paper we present an adaptive mechanism that enables a normative framework to change deterrence sanctions according to an agent population, in order to preclude agents from exploiting potential normative flaws. The system tries to avoid institutional control beyond what is strictly necessary, seeking to maximize agent contracting activity while ensuring a certain commitment compliance level, when agents have unknown risk and social attitudes.
\end{abstract}

\section{Introduction}

Multi-agent systems applied to B2B have been widely studied (e.g. [1, 2]). Interaction infrastructures for autonomous agents representing real-world business entities (such as enterprises) have been developed (e.g. $[3,4]$ ), including negotiation and contracting facilities. Normative environments are middleware that provide support for making agents' mutual commitments explicit. Those commitments are expressed as norms (behavior prescription rules), which can be assembled in contracts. Furthermore, when embedded in some notion of "institution", normative environments take an active role in checking agents' compliance with their commitments. As such, electronic institutions $[5,6]$ have been developed with norm monitoring and enforcement facilities in place, with the aim of establishing trust among participants in a normregulated relationship, giving contracts a binding force.

Another important facet of an electronic institution is its ability to facilitate contract establishment, by providing a normative framework [6]. This way, agents need not specify all norms applicable to their contracts, but may instead rely on a normative background. This feature is especially important when considering contrary-to-duty situations, which typically should not be likely to occur. A contract's normative structure will certainly reflect the coarse business workflow between the involved agents, but will probably include provisions for the most likely possible violations only. Further contingencies will often not be dealt with when establishing a contract, because it may be costly or even impossible to anticipate them.

Normative environments can provide trust by using different enforcement mechanisms. One such mechanism consists of making sure that norms are applied as defined in a contractual relationship. Some of those norms will state what agents should do when they violate some obligation (e.g., if an agent fails to deliver a good that has been promised, he should return the payment he has received with a $10 \%$ increase). These norms are known as contrary-to-duties. However, as mentioned above, in certain cases there will be no specified consequence. This is when other coercive approaches may be relevant, in situations where agents try to take advantage of their potential gain when violating norms (because they might be more self-interested than socially concerned).

In the literature (e.g. [7, 8]) we find, among others, two basic kinds of sanctions that an institution may apply in order to incentive norm compliance (or, to put it another way, to discourage deviations). Direct material sanctions have an immediate effect, and consist of affecting the resources an agent has (e.g. by applying fines). Indirect social sanctions, such as changing an agent's reputation, may have an effect that extends through time. Depending on the domain and on the set of agents that inhabit the institutional environment, the effectiveness of such sanctions may be different: if agents are not able to take advantage of other agents' reputation information, material sanctions should be used insteadhnology 
There are two general policies used when applying (direct) sanctions, which concern their intended effects: (i) deterrence aims at punishing the violator so as to discourage future violations; (ii) retribution aims at compensating the addressee of the violation. Bringing these policies to the electronic institution realm, we consider that retribution sanctions are those specified in contractual norms, be they negotiated or inherited from a preexistent normative framework. In this case the institution, while monitoring norm compliance, acts as a mediator. As for deterrence sanctions, they will be applied by the institution itself, and may be used so as to maintain order (by motivating agents to comply) and consequently trust in the system. A similar distinction is made in [9], where active sanctions describe actions to be performed by the violator (and if he does so the violation will become extinguished) and passive sanctions describe actions that the norm enforcer is authorized to perform.

Economic approaches to law enforcement have suggested analyzing sanctions and their amplitude by taking into account their effects on parties' activities. Agents committing to norms that have associated deterrence sanctions enter risky activities, because they may unintentionally violate them. It has been argued [10] that under strict liability (where violators are always sanctioned) sanctions should equal harm done. An increase in the level of activity brings an increase in the expected harm; if damages equal harm, parties will have socially correct incentives to engage in risky activities (that is, to establish commitments). However, this conclusion relies on the additional assumption that parties are risk-neutral. If they are risk-averse, the optimal level of damages tends to be lower than harm. This comes from the fact that with risk-aversion, a sanction imposes a cost which does not exist under risk neutrality. As explained in [11], risk-aversion introduces costless deterrence and the policy-maker (an electronic institution in our case) should take that into account when choosing the optimal sanction.

The presence of social sanctions will also influence the behavior of agents concerning their commitments. Reputation-aware environments should have a lesser need for deterrence sanctions (see, for instance, [12]). Besides reducing agents' risk (see above), this reduction in deterrence measures may be important for other reasons. On one hand, both the enforcement activities and the completion of direct sanctions may be costly, which asks for either lowering the resources used in those activities or eliminating sanctions in noncompensating cases. On the other hand, we can imagine (at least in theory) a computational system where these costs can be marginal: assuming that automatic norm monitoring is computationally inexpensive and that sanctions consist e.g. of fines that are debited from agents' accounts administered by the system. But in this case, higher fine levels require higher financial warranties from agents, which may once again decrease their level of activity: some agents may not meet such requirements, which will inhibit them from committing to certain normative relationships.

In this paper we seek to explore these issues inside an institutional environment, under the following assumptions:

- Strict liability: a norm violation is always detected.

- Costless enforcement: monitoring and sanctioning have a negligible cost to the institution.

- Unknown agent population: concerning agents' risk tolerance and social awareness.

We envisage a normative framework that is able to adapt itself (by changing applicable sanctions) according to some measurement of success, which will have to manage the following conflicting goals: i) keep the normative framework as simple as possible, by avoiding over-constraining the environment; and ii) maximize trust on the institutional environment's use. These conflicting goals must be balanced well enough in order to encourage agents to increase their level of activity, when the agent population's risk tolerance is unknown beforehand.

In the following section we present an abstract model for contractual commitments and the adaptation approach that we take. Section 3 describes a simulation environment, and specifies the adaptation approach by describing how it is implemented and tested. Section 4 presents some experiments. Section 5 concludes and describes related work.

\section{The model}

In our approach we take the stance that agents are truly autonomous, and thus cannot be forced to fulfill their obligations. The institution may, however, impose certain fines as deterrence sanctions: those fines are assumed to be fully regimented (that is, agents cannot escape them, e.g. because they were required, upon entering the institution, to make a deposit that is in control of the institution). Sanctions other than fines could also be envisaged as deterrence measures.

We are mainly concerned with contracting scenarios, wherein agents make mutual commitments and create business expectations. Violations, even when handled by contractual norms, should be seen as exceptional situations. Hence, if a certain kind of violation becomes frequent, response should be taken through an increase of sanctions. 


\subsection{Commitment trees}

In order to obtain a tractable model for handling contractual commitments, we use a tree-based representation for interdependent obligations. This representation is useful for understanding the simulation model that we describe later on.

When establishing contracts, agents create a network of directed obligations, some of which are dependent on the fulfillment or violation of other obligations. Consider the following two-party contract: agent $a$ will pay $p$ units to agent $b$, after which $b$ will deliver $x$ to $a$. In case $b$ fails to deliver, he must return $p^{\prime}=p+\delta$ to $b$. This sequence of commitments is illustrated in Figure 1, in a tree-like structure - a commitment tree. Each node represents an obligation, and each labeled directed edge indicates, in the child node pointed to, what follows when the obligation contained in the parent node is fulfilled (Fulf) or violated ( $\mathrm{Viol})$. Note that in this simple example nothing is specified yet if agent $a$ violates his commitment to pay $p$, nor if agent $b$ violates his commitment to return $p$ '.

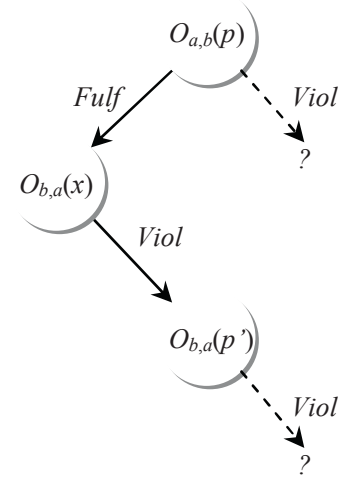

Figure 1. Sample commitment tree

While this example shows a simple binary tree, one can imagine multi-party contracts with a potentially complex commitment tree structure ${ }^{1}$.

The violation of an obligation with a prescribed sanction may simply denote a case where an agent preferred to incur the prescribed sanctions for matters of conflicting goals (e.g. he had another contract which

\footnotetext{
1 The tree will not be binary if each obligation fulfillment or violation may lead to more than one consequence. Also, if we consider that a norm can prescribe an obligation if two or more fulfillments or violations occur, we end-up with a directed acyclic graph instead of a tree, since each node may have more than one parent. However, this is not very common in the case of violations (which are our main concern here): each violation will typically be handled in isolation (as in the model of "reparation chains" in [13]).
}

was more important than this one, and could not stand for both). If such violation becomes frequent, however, this may denote a flaw in the normative system that agents are being able to exploit to their own advantage.

\subsection{Adaptation}

The importance of adaptation in a normative framework resides in the fact that contracts may be unfair in certain execution outcomes. If self-interested agents explore such flaws to their own profit, action should be taken in order to discourage such behaviors.

In order to build a model that adapts the normative framework in a domain-independent way, we will concentrate on adding deterrence fines to the system (which are not violable), instead of changing the prescribed obligations in each violation situation. The normative framework's adaptation is based on associating, with each obligation, a fine that can be strengthened or weakened (see Figure 2). With this approach, every obligation will have a (potentially null) fine to be imposed on the bearer in case of violation; this fine is added up to the violation consequence in the child node already in the tree, if there is one.

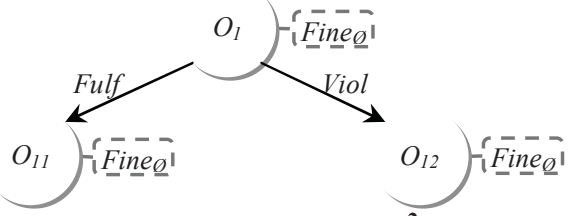

Figure 2. Binary commitment tree ${ }^{2}$ with null fines

In order to correctly model appropriate responses to specific situations, we need to assess how often an obligation is used, and how often it is violated. Fines will be updated according to these measurements. The basic principle that we rely on is that the strength of a fine should be directly proportional to its application frequency. As such, fines should increase when they are applied often, and decrease when they are not used. A low level of fine usage indicates that obligations are being fulfilled or they are not being used as often as desired: in both cases fines should be decreased, since they either are not needed or are inhibiting activity. On the other hand, a high level of fine usage means that agents still prefer to go through the sanction, and as such it should be increased as a deterrence mechanism. In summary, this approach tries to make fines (a) strong enough to discourage deviation and (b) weak enough to avoid unnecessary institutional control.

2 From now on, we will only consider the case for binary commitment trees (this simplification does not limit the applicability of our approach, while it does make it easier to follow). 


\section{Simulation environment}

Aiming at the development of a simulation prototype that allows us to test the adaptation model briefly described above, we designed the following experimental scenario.

A number of agents will be in the system, and each will be given the opportunity to sign a contract. The contract's structure is defined by the number of enacting roles and by an underlying binary commitment tree (BCT from now on). Roles are used as bearers or counterparties of obligations in the tree. Furthermore, each obligation has an associated cost (to be supported by a fulfilling bearer) and benefit (to be collected by the counterparty of a fulfilled obligation). Figure 3 summarizes the characterization of a commitment.

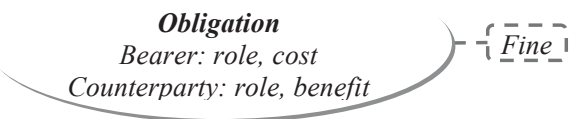

Figure 3. A node in a BCT and its configuration

When an agent decides to sign a contract, he will enact the corresponding commitment tree with a role assigned to him before contracting. We say that the state of a contract enactment is the commitment currently under appreciation. If the bearer of such a commitment is the agent that decided to contract, he will be asked for a play: either to fulfill or to violate such a commitment. If the commitment's bearer is not the agent, the system will decide whether the commitment will be fulfilled or not, according to a uniform strategy. The current state will be updated according to the decision taken: if the choice is to fulfill, the state will become the root commitment of the fulfillment sub-tree; if the choice is to violate, the state will become the root commitment of the violation sub-tree. The contract terminates when the state reaches a null value (i.e. when no fulfillment/violation sub-tree exists upon a fulfill/violate decision).

\subsection{Agent decision-making}

Each agent has two distinct kinds of decisions to make. If he does not currently have an ongoing contract, he is given the opportunity to sign one. For that, a random role from the contract structure is selected and the agent is asked if he wants to contract with that role. Each agent is configured with a risk tolerance parameter $R t \in[0 ; 1[$, which denotes his willingness to contract in the presence of violation fines. If $R t=0$, the agent will only decide to contract if he will be subject to no fines at all. On the other extreme, if $R t \approx 1$, the agent will always risk to contract, regardless of any fines. An agent will decide to contract depending on the highest fine that is associated with commitments for the assigned role. In order to contract, the following relation should be true:

$$
\text { highestFine (role) } \leq b * R t /(1-R t)
$$

where $b$ is a slope parameter associated with the agent's budget.

We assume that agents always prefer to contract, regardless of commitment costs or benefits. A contract is presumably beneficial to all partners should they fulfill all their commitments. Having said this, we allow a contract to be unbalanced or incorrect from a safeness point of view, in the sense discussed in [2]. In our case, we consent that participating in the contract may in some cases be worse-off than not participating, depending on the behavior of contractual partners.

When an agent has an ongoing contract, whenever the contract's state is a commitment where he is the bearer he will decide whether to fulfill or to violate such a commitment. Depending on a so-called incontract strategy, the agent will explore the contract's BCT in order to decide which option is best for him. Such strategies may vary from simply comparing the cost of fulfillment with the applicable fine in case of violation, to computing the path with the best outcome from the whole BCT. Some possible strategies will be presented in section 4.

Agents are essentially expected utility maximizers. This means that, in principle, they will fulfill obligations only when the expected outcome from this choice is better than the expected outcome from violating (according to his in-contract strategy). We do however embed in our agents some notion of social welfare, which impels them to fulfill even when they do not have a strict advantage in doing so. While for now we do not consider the effect of reputation in future contracts, we allow in our model that agents are not all equally self-interested. For that we introduce a social awareness parameter $S a \in[0 ; 1[$. If $S a=0$, the agent will violate whenever the outcome from this choice is better than the outcome from fulfilling. On the other extreme, if $S a \approx 1$ the agent will always choose to fulfill. The agent will decide to fulfill an obligation $o$ whenever the following relation is true:

$$
\begin{gathered}
\text { violationOutcome }(o)-\text { fulfillmentOutcome }(o) \leq \\
b * S a /(1-S a)
\end{gathered}
$$

where $b$ is as before. The violation/fulfillment outcomes are calculated by the in-contract strategy. 


\subsection{Fine update function}

In each simulation step, all agents running in the simulation will have a chance to play. After this, the contract structure will have a chance to adapt, that is, the fines associated to the BCT will be updated. Each fine is updated independently of all other fines.

In order to delineate a fine update policy, we first need to define the goal function that will be pursued. As mentioned before, fine updates should take into account how often they are applied. We define a threshold parameter $T h \in[0 ; 1]$ that indicates the highest percentage of fines that the system should accept as normal. For instance, with a value $T h=0.1$ we are saying that if more than $10 \%$ of the agents running in the simulation violate a given obligation the normative system will raise the fine in the next step - in this case, we say that $10 \%$ of the total number of agents is the number of tolerated violations. Furthermore, since not all agents will be in the same state at a given time point, we adjust the threshold according to the number of agents that did in fact make a decision concerning the fulfillment or violation of a specific obligation (because they were in that state). For instance, if with a group of 1000 agents we have 10 violations of a specific obligation in a simulation step, this may have a different response from the normative framework depending on the number of agents that went through that same obligation at that time step. If there were 10 play decisions taken on that obligation, this makes a $100 \%$ percentage of violations; if there were 100 plays, that percentage comes down to $10 \%$. While in none of these cases we exceed $10 \%$ of the total number of agents (1000), it seems clear that the system should react on the former case.

A state's fine will be increased if the number of violations exceeds the following tolerated violations function:

$$
\begin{gathered}
\text { toleratedViolations }= \\
2 * \text { Th*Nag } /\left(1+e^{-(5 / \text { Nag }) * x}\right)-T h * N a g
\end{gathered}
$$

where Nag is the number of agents running in the simulation and $x$ is the number of agents that were in this state. This is a sigmoid function with an upper bound set at $T h * N a g$ (a percentage of the total number of agents). The steepness parameter is $5 / \mathrm{Nag}$, which makes the sigmoid curve approach the upper bound close to Nag, which is the ceiling for $x$ (there can be no more than Nag agents at this state).

If the number of violations does not exceed the number of tolerated violations, then the fine will be decreased. Fines are increased heavier than they are decreased. We have set an increase step of 0.1 and a decrease step of 0.01 . Fines will be applied rounded to the first decimal place, which gives a sense that it takes ten simulation steps (without exceeding the tolerated violations function) to decrease the fine value.

\section{Experiments}

In this section we describe some experiments made using the described simulation environment and model.

\subsection{Experimental settings}

What we want to study with the simulation scenario described in the preceding section is whether the normative framework is able to adapt and stabilize fine changes in a situation with a static agent population. Furthermore, the system should keep fines as low as possible, while still conforming to the goal function outlined above. This is because the system aims to avoid excessive control and through that maximize agents' contracting activity, which should be obtained with less risk exposure in an agent population with unknown risk tolerance.

If we change the agent population in the middle of the simulation, then we have a moving target setting, which is out of the scope of the experiments reported in this paper. However, since we lower fines whenever the tolerated violations are not exceeded, we believe that the system will quickly adapt in a moving target setting.

4.1.1. Contract structures. Since we are not concerned with the correctness of the contract to be signed, we may experiment with a large number of different BCTs. Figure 4 shows some possibilities, all considering two roles only. For instance, (d) includes two complementary obligations 0 and 1 , and their respective contrary-to-duties 3 and 2. We shall call obligation 1 the to-duty obligation of obligation 0 .

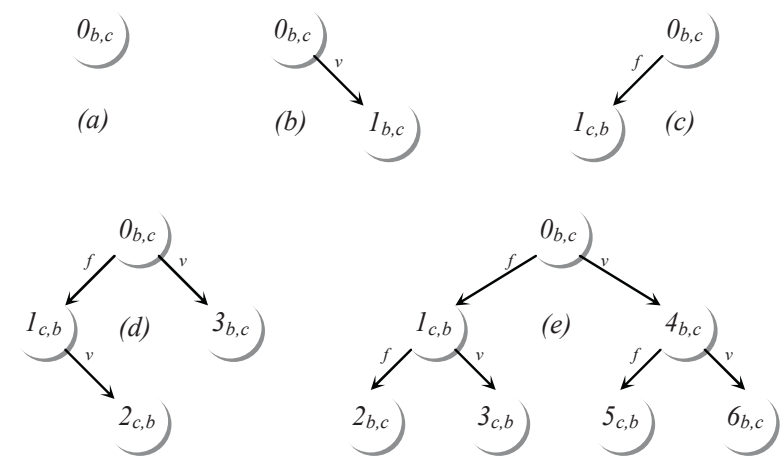

Figure 4. Binary commitment trees: each node $I d_{i, j}$ is an obligation, where $i$ is the bearer and $j$ is the counterparty 
We will present some experimental results based on some of these BCTs. In all cases, obligation costs were set at 10.0 and benefits at 12.0 (setting benefits higher than costs tries to give all partners some gain when the contract is well-balanced). Also, fines were initialized at 0.0 .

4.1.2. Agents. As mentioned before, we aim at testing the normative framework's adaptation when the agent population is unknown, concerning agents' risk tolerance and social awareness. For that reason, all agents in the system have a uniform random distribution concerning the risk-tolerance and socialawareness parameters. Also, concerning the use of these parameters the slope value $b$ was set to 10.0 .

Several in-contract strategies can be devised, representing the reasoning abilities of agents when deciding whether to fulfill or violate an obligation. As explained in section 3.1, the in-contract strategy will be used to compute the fulfillment and violation outcomes at a given state. We consider the following simple strategies, which may have different relevance depending on the BCT at hand:

i. Local: fulfillment cost vs. fine

ii. LocalCtd: fulfillment cost vs. fine + contraryto-duty cost (ignore possible entitlements in case of fulfillment)

iii. LocalTd: fulfillment cost + to-duty benefit vs. fine (ignore possible normative sanctions in case of violation)

iv. LocalBoth: fulfillment cost + to-duty benefit vs. fine + contrary-to-duty cost

v. FulfillmentBalance: balance if every participant fulfills the contract vs. fine

vi. DoubleFulfillmentBalance: balance if every participant fulfills the contract vs. balance if every participant fulfills the contract from the contrary-to-duty obligation onwards - fine

vii. BestPathCompliantPartners: best balance for every path in the BCT, assuming that contract partners always fulfill

viii. BestPathMinimax: best balance for every possible path in the commitment tree, considering that contract partners will use the same strategy

Strategies iii through vii assume that partners will always fulfill their obligations. Analyzing these strategies together with the BCTs depicted in Figure 4, we can see that, for instance, FulfillmentBalance will only make sense in tree (e), since in all other BCTs the same outcome can be achieved with less computationally demanding strategies.

\subsection{Experiments and results}

In all experiments a uniform strategy "always fulfill" was used by the system for commitments whose bearer is not a simulation agent. The violation threshold parameter Th was set to 0.1. Each simulation was run with 10000 agents and 1000 time steps.

Figures 5-9 present the results of fine evolution in some possible combinations of BCTs and in-contract strategies. In BCT (c) a LocalTD strategy (Figure 5) is able to grab the benefit achieved from obligation 1 when fulfilling obligation 0 . Only the violation of obligation 1 is tempting, and thus the system adapted the corresponding fine.

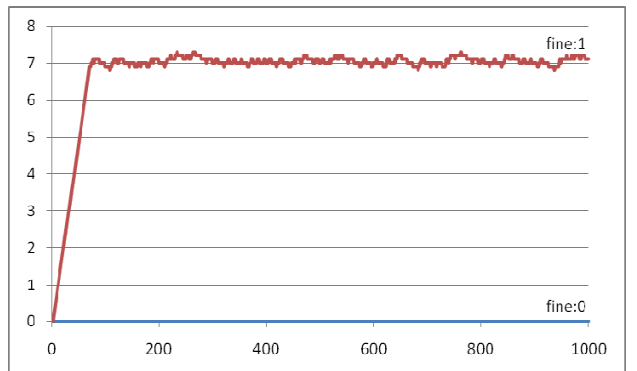

Figure 5. Fine evolution for BCT (c) and LocalTd

In BCT (d) the LocalTD strategy (Figure 6) impels agents to fulfill obligation 0 . Agents that are more socially concerned will tend to fulfill obligation 1 with lower fines than other agents, hence the difference between fines 1 and 2 . In the same scenario, BestPathMinimax (Figure 7) gives agents the ability of evaluating every possible outcome with rational plays from both contractual partners. Each agent playing in state 0 initially violates because he sees his partner preferring to violate obligation 1 (and 2), therefore giving him no benefit. However, when fines 2 and 3 are high enough, fines 0 and 1 are no longer necessary.

As for BCT (e), unlike the previous two contractual structures, this one is not profitable (with the complete fulfillment execution 0-1-2) for the agent playing at state 0 , if we consider the values set for every obligation's cost (10.0) and benefit (12.0). The DoubleFulfillmentBalance strategy (Figure 8) is able to detect the better path $0-4-5$, causing a reaction of the normative system with a raise of fine 0 . Without this escape, violating obligation 2 is a means of taking some profit out of the game, bringing a raise of fine 2 . The BestPathMinimax strategy in this scenario (Figure 9) makes up the most complex setting we have experimented. Starting at state 0, the best path when playing with a similar agent is initially to violate all obligations (including contrary-to-duties), bringing an outcome of 0 . This is because the agent assumes that 
his partner will maximize his own profit, therefore preferring to violate at states 1 and 5. Fines at obligations 0 and 1 become ineffective as soon as fines associated with contrary-to-duties are high enough.

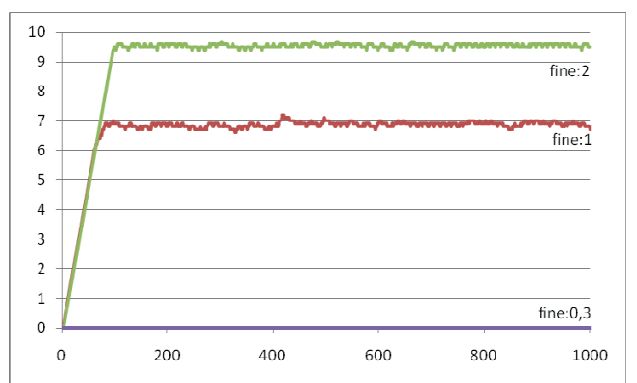

Figure 6. Fine evolution for BCT (d) and LocalTd

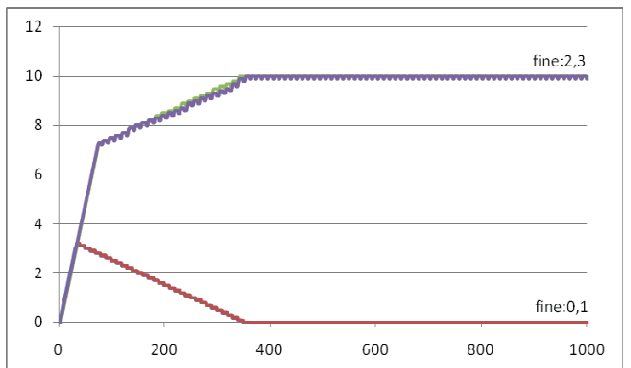

Figure 7. Fine evolution for BCT (d) and BestPathMinimax

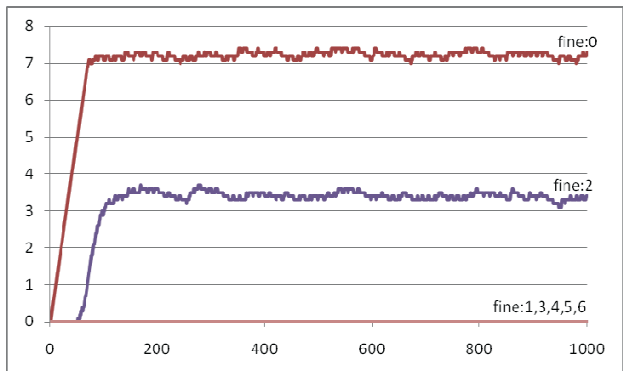

Figure 8. Fine evolution for BCT (e) and DoubleFulfillmentBalance

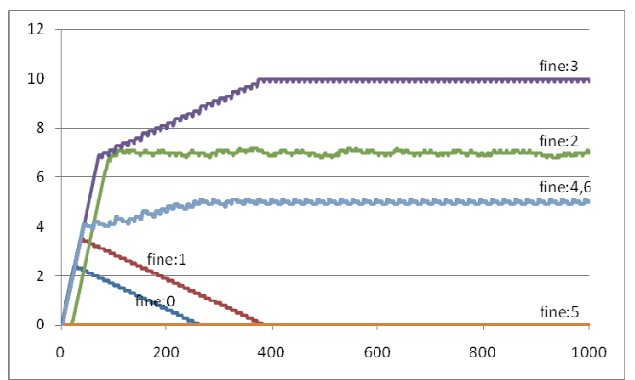

Figure 9. Fine evolution for BCT (e) and BestPathMinimax
With any combination of BCTs and in-contract strategies the system is able to adjust the deterrence sanction values to the behavior of an agent population, stabilizing fines after a period of time. We should emphasize that the system continuously tries to lower fines, which is observable by the slight fluctuations of fines towards the end of the curves in figures 5-9. Therefore, system imposed fine levels are the lowest that keep violations below the tolerated violations function.

\section{Conclusions and related work}

In this paper we have presented a simple model for the adaptation of deterrence sanctions used in a normative framework. We have shown that it is feasible to adapt deterrence levels to the behavior of an agent population. This is important when the normative space has imperfections that make the contracts to which norms apply unfair, opening the possibility for selfinterested agents to exploit their potential advantage.

We have built an abstraction for contractual commitments by modeling their corresponding obligations in a binary commitment tree structure. In such a tree we are able to include both "to-duty" complementary obligations and contrary-to-duty retribution sanctions.

The parameterization of agents with different social attitudes is common in computational models for social interactions. Agents range from selfish to respectful [14]. Respectful agents are those that internalize norms and fulfill obligations simply because they are obligations [15], irrelevant of there being associated sanctions in case of violation. Our social awareness parameter tries to take this heterogeneity of social attitudes into account.

Influencing agent decision making regarding social commitments is generally conceived as social control [15], and is usually focused on enforcement, sanctions and reputation. A different perspective has been taken in [16], where some agents in the system are directly controlled by the system's designer. Making such agents play specific strategies will lower the payoff of joint activities when uncontrolled agents play selfishly, therefore making them choose to fulfill. This seems unrealistic in contracting scenarios. Yet, the authors have made a theoretical analysis in scenarios where uncontrolled agents are expected utility maximizers and when they are reinforcement learners. Such scenarios can be tested in our simulation model as well.

Dynamic properties of normative systems have been studied from different perspectives. In [17] norms are seen as patterns of behavior that may emerge bottom- 
up from agent interactions. In our case, however, the normative system is external to the agents, and we seek to adapt it to a specific agent population in order to pursue an overall system goal.

Self-adaptation of institutional normative environments is also studied in [18], with two significant differences to our approach. First, their adaptation model is based on the definition of domaindependent transition functions, stating what specific change should be made in a specific norm when some goal specification is not met. Second, their model does not assume strict liability; therefore, agents are able to violate norms while not being detected.

In this paper we have not considered the influence of reputation on agent's contractual behavior. It has been argued [12] that in the presence of reputation mechanisms there is a lesser need for deterrence policies. We believe that positive reputation updates triggered by the normative environment may be an incentive for agents to fulfill their commitments.

Our simulation environment opens up the possibility for several studies, such as the impact of changing risktolerance and social-awareness parameter distributions in the value of imposed fines [19]. We have shown that under uniform random distributions the system is able to adapt by appropriately raising and stabilizing fine values.

\section{Acknowledgments}

The first author is supported by FCT (Fundação para a Ciência e a Tecnologia), Portugal, under grant SFRH/BD/29773/2006.

\section{References}

1. Blake, M.B., Agent-oriented approaches to $B 2 B$ interoperability. The Knowledge Engineering Review, 2001. 16(4): p. 383-388.

2. Desai, N., N.C. Narendra, and M.P. Singh. Checking correctness of business contracts via commitments. in Proceedings of the 7th international joint conference on Autonomous agents and multiagent systems. 2008. Estoril, Portugal: IFAAMAS.

3. Sallé, M., Electronic Contract Framework for Contractual Agents, in Advances in Artificial Intelligence: 15th Conference of the Canadian Society for Computational Studies of Intelligence, R. Cohen and B. Spencer, Editors. 2002, Springer. p. 349-353.

4. Lopes Cardoso, H. and E. Oliveira, Electronic Institutions for B2B: Dynamic Normative Environments. Artificial Intelligence and Law, 2008. 16(1): p. 107-128.

5. García-Camino, A., et al., Norm-Oriented Programming of Electronic Institutions: A Rule-Based Approach, in
Coordination, Organizations, Institutions, and Norms in Agent Systems II, P. Noriega, et al., Editors. 2007, Springer. p. 177-193.

6. Lopes Cardoso, H. and E. Oliveira, A Context-based Institutional Normative Environment, in Coordination, Organizations, Institutions, and Norms in Agent Systems $I V$, J. Hubner, et al., Editors. 2009, Springer. p. 140-155.

7. Pasquier, P., R.A. Flores, and B. Chaib-Draa, Modelling Flexible Social Commitments and their Enforcement, in Engineering Societies in the Agents World V, M.-P. Gleizes, A. Omicini, and F. Zambonelli, Editors. 2005, Springer: Toulouse, France. p. 139-151.

8. Grossi, D., H. Aldewereld, and F. Dignum, Ubi lex, ibi poena: Designing norm enforcement in e-institutions, in Coordination, Organizations, Institutions, and Norms in Agent Systems II, P. Noriega, et al., Editors. 2007, Springer. p. 101-114.

9. Fornara, N. and M. Colombetti. Specifying and Enforcing Norms in Artificial Institutions. in 7th Int. Conf. on Autonomous Agents and Multiagent Systems (AAMAS 2008). 2008. Estoril, Portugal.

10. Polinsky, A.M. and S. Shavell, Punitive Damages, in The New Palgrave Dictionary of Economics and the Law, P. Newman, Editor. 1998, Stockton Press: New York. p. 192-198.

11. Garoupa, N., The Theory of Optimal Law Enforcement. Journal of Economic Surveys, 1997. 11(3): p. 267-295.

12. Luck, M., et al., Trust and Norms for Interaction, in Proceedings of the IEEE SMC 2004 International Conference on Systems, Man \& Cybernetics. 2004, IEEE: The Hague, The Netherlands. p. 1944-1949.

13. Governatori, G. and A. Rotolo, Logic of Violations: A Gentzen System for Reasoning with Contrary-To-Duty Obligations. Australasian Journal of Logic, 2006. 4: p. 193-215.

14. Boella, G. and L. van der Torre. A Game-Theoretic Approach to Normative Multi-Agent Systems. in Normative Multi-agent Systems. 2007: Schloss Dagstuhl.

15. Castelfranchi, C., Engineering Social Order, in Engineering Societies in the Agents World, A. Omicini, R. Tolksdorf, and F. Zambonelli, Editors. 2000, Springer: Berlin, Germany. p. 1-18.

16. Brafman, R.I. and M. Tennenholtz, On Partially Controlled Multi-Agent Systems. Artificial Intelligence Research, 1996. 4: p. 477-507.

17. Sen, S. and S. Airiau. Emergence of norms through social learning. in 20th International Joint Conference on Artificial Intelligence. 2007. Hyderabad, India.

18. Campos, J., et al., Formalising Situatedness and Adaptation in Electronic Institutions, in Coordination, Organizations, Institutions, and Norms in Agent Systems $I V$, J. Hubner, et al., Editors. 2009, Springer. p. 126-139.

19. Lopes Cardoso, H. and E. Oliveira, Risk Tolerance and Social Awareness: Adapting Deterrence Sanctions to Agent Populations, in 14th Portuguese Conference on Artificial Intelligence (EPIA 2009). 2009, Springer (to appear): Aveiro, Portugal. 